\title{
Boundaries and Prototypes in Categorizing Direction
}

Mast, V.; Wolter, D.; Klippel, A.; Wallgrun, J.O.; Tenbrink, T.; Freksa, C.; Nebel, B.; Hegarty, M.; Barkowsky, T.

\section{Spatial Cognition IX}

\author{
DOI: \\ 10.1007/978-3-319-11215-2_7
}

Published: 15/09/2014

Peer reviewed version

Cyswllt i'r cyhoeddiad / Link to publication

Dyfyniad o'r fersiwn a gyhoeddwyd / Citation for published version (APA):

Mast, V., Wolter, D., Klippel, A., Wallgrun, J. O., Tenbrink, T., Freksa, C. (Ed.), Nebel, B. (Ed.), Hegarty, M. (Ed.), \& Barkowsky, T. (Ed.) (2014). Boundaries and Prototypes in Categorizing Direction. In Spatial Cognition IX: Lecturers Notes in Computer Science (2014 ed., pp. 92-107). Springer. https://doi.org/10.1007/978-3-319-11215-2_7

\footnotetext{
Hawliau Cyffredinol / General rights

Copyright and moral rights for the publications made accessible in the public portal are retained by the authors and/or other copyright owners and it is a condition of accessing publications that users recognise and abide by the legal requirements associated with these rights.

- Users may download and print one copy of any publication from the public portal for the purpose of private study or research.

- You may not further distribute the material or use it for any profit-making activity or commercial gain

- You may freely distribute the URL identifying the publication in the public portal ?
}

Take down policy

If you believe that this document breaches copyright please contact us providing details, and we will remove access to the work immediately and investigate your claim. 


\title{
Boundaries and Prototypes in Categorizing Direction
}

\author{
Vivien Mast ${ }^{1}$, Diedrich Wolter ${ }^{2}$, Alexander Klippel ${ }^{3}$, Jan Oliver Wallgrün ${ }^{3}$, and \\ Thora Tenbrink ${ }^{1,4}$ \\ 1 I5-[DiaSpace], SFB/TR 8 Spatial Cognition, University of Bremen \\ viv@informatik. uni-bremen.de \\ 2 Smart Environments, University of Bamberg \\ diedrich.wolter@uni-bamberg.de \\ 3 GeoVISTA Center, Department of Geography, The Pennsylvania State University \\ $\{k l i p p e l$, wallgrun\}@psu.edu \\ 4 School of Linguistics and English Language, Bangor University, Wales, UK \\ t.tenbrink@bangor.ac.uk
}

\begin{abstract}
Projective terms such as left, right, front, back are conceptually interesting due to their flexibility of contextual usage and their central relevance to human spatial cognition. Their default acceptability areas are well known, with prototypical axes representing their most central usage and decreasing acceptability away from the axes. Previous research has shown these axes to be boundaries in certain non-linguistic tasks, indicating an inverse relationship between linguistic and non-linguistic direction concepts under specific circumstances. Given this striking mismatch, our study asks how such inverse non-linguistic concepts are represented in language, as well as how people describe their categorization. Our findings highlight two distinct grouping strategies reminiscent of theories of human categorization: prototype based or boundary based. These lead to different linguistic as well as non-linguistic patterns.
\end{abstract}

\section{Introduction}

Imagine you want to divide a circle into four segments, on a piece of paper. A natural solution would be to cut it into quadrants using a vertical and a horizontal stroke of the pen. This segmentation is easy to do and to think about, but surprisingly hard to put into words. Simple direction concepts such as left and right just do not fit very well. However, other ways of categorizing space-for example, based on these simple direction concepts - may serve as natural solutions in other contexts. In this paper, we ask how people categorize direction and how they verbalize these categories, relevant to long-standing debates about human categorization and the relationship between language and thought.

Categorization has been a topic of central interest in psychology and linguistics for many decades (e.g., Smith and Medin, 1981; Taylor, 1989). In the 1970ies, a major paradigm shift led away from previous assumptions that humans distinguish members from non-members of a category by means of essential features (boundary-based categorization). Categories are now typically seen as 
characterized by an idealization of what a perfect member would be, with membership depending on overall perceived similarity to this prototype (Mervis and Rosch, 1981).

In much of the earlier research on categorization, linguistic expressions and non-linguistic concepts were not distinguished very clearly. However, in a different strand of research, the linguistic relativism debate initiated by Whorf (1956) is aimed at determining the extent and directionality of correspondences between language and thought (Bierwisch and Schreuder, 1992, Levinson et al., 2002 ; $\mathrm{Li}$ and Gleitman, 2002). With respect to spatial directions, this relationship has been subject to debate, as we will outline next.

\section{Spatial Relations and their Categorization}

It is uncontroversial that the use of projective terms such as left, front, above relies heavily on prototypical axes: turning right invokes a prototypical $90^{\circ}$ angle from a view direction just as well as reference to an object on your right does (Tenbrink, 2011), with various linguistic effects when the relevant direction departs from the prototypical axis (Gapp, 1995, Logan and Sadler, 1996, Tenbrink, 2007, Zimmer et al. 1998). While systematic differences emerge depending on the task setting (Vorwerg and Tenbrink, 2007), in particular between dynamic and static uses of projective terms (Herzog, 1995, Hickmann and Hendriks, 2006), existing research shows a stable prototype effect amongst a broad range of tasks.

Regarding the relation to non-linguistic categories, however, the picture is less clear. While Hayward and Tarr (1995) claim that both verbal and non-verbal categories have the same prototype structure, there is compelling evidence that linguistic prototypes for horizontal and vertical directions can correspond to boundaries in non-linguistic categorization (Crawford et al., 2000; Huttenlocher et al., 1991; Klippel and Montello, 2007). This may be due to differences in the specific task and analysis procedures. Hayward and Tarr (1995) showed participants images with configurations of schematic objects. Participants replicated the position of the locatum on a sheet of paper showing only the relatum, or judged the similarity of two consecutively shown configurations. In both tasks, accuracy was greatest when the locatum was on a perpendicular axis. From this, Hayward and Tarr (1995) conclude that verbal and non-verbal categories have the same prototype structure. In contrast, Crawford et al. (2000) and Huttenlocher et al. (1991) presented experiments addressing estimation bias effects in memory tasks. Studies in a large range of domains have demonstrated that humans show an estimation bias away from category boundaries towards prototypes in memory reproduction tasks (Crawford et al., 2000), a fact which can be exploited for analyzing the conceptual structure of human categories. When reproducing the position of a dot in a circle (Huttenlocher et al. 1991), or of a dot in relation to a schematic image of a television (Crawford et al., 2000), participants showed an angular bias of the reproduction towards the center of quadrants, away from the perpendicular axes. Linguistic ratings on the same stimuli, however, show the typical prototype effect. Thus, Crawford et al. (2000) conclude that the 
perpendicular axes play the role of prototypes for linguistic categories while they serve as boundaries for non-linguistic categories.

Further evidence supporting this surprising claim was subsequently obtained by Klippel and Montello (2007), who found that linguistic awareness influences categorization of turn direction concepts in a similar way. Participants were presented with depictions of turns of different angles and asked to group them into categories based on similarity - first without, then with the knowledge that they would later be asked to label the groups linguistically. Klippel and Montello (2007) identified the items which were most consistently categorized together using a cluster analysis. Without verbal task awareness, the aggregated categories of all participants had the perpendicular right and left axes as boundaries. When participants were aware of the verbal task, the aggregated categories had the perpendicular axes in their center, suggesting a prototype structure.

These findings are highly relevant for both of the debates mentioned above. With respect to the controversy on linguistic relativism, the findings suggest that language and cognition may sometimes diverge. On the other hand, the debate on boundary vs. prototype based categorization may have to be addressed separately for linguistic as opposed to non-linguistic categories.

These findings are furthermore relevant when considering the discrepancy between concept-based knowledge representation approaches such as spatial calculi and psycholinguistic research. Spatial calculi typically treat projective terms as crisp, mutually exclusive categories that allow for feasible reasoning processes (Renz and Nebel, 2007), contrasting with the psycholinguistic findings on gradedness of spatial concepts. Shedding light on these issues may improve the further development of separate, but interconnected conceptual vs. linguistic ontologies (Bateman et al. 2007) by gaining a better understanding of how a cognitively adequate mapping between the different ontologies may be achieved.

To our knowledge, only Klippel and Montello (2007) have used a grouping task for a non-linguistic categorization assessment of spatial directions. Although their analysis reveals a difference between linguistic and non-linguistic grouping patterns similar to the results by Crawford et al. (2000), Klippel and Montello (2007) do not assess the role the axes played in the participants' conceptualizations of the groups. Also, they address (dynamic) turn direction concepts rather than (static) spatial relationships. With respect to the spatial relation between two locations, the nature of the relationship between linguistic and non-linguistic categories has only been tested on memory tasks.

Moreover, the task settings so far have all been based on schematic depictions, with dots and lines representing spatial directions. Real world relationships between objects are far more complex, often involving extended shapes and functional relationships that lead to distinct effects on verbalization (Carlson and van der Zee, 2005, Zwarts, 2003). It is unknown, so far, if more realistic scenes would lead to similar categorization patterns as found in the abstract settings.

Finally, although a range of findings exist with respect to the verbalization of non-prototypical spatial relationships, no studies so far have addressed systematically just how non-linguistic categories that do not directly correspond to linguistic categories are referred to in language. How will the quadrants in a 
segmented circle be described? While previous findings show that non-typical spatial relationships lead to more complex spatial expressions (Tenbrink, 2007, Zimmer et al. 1998), these only concern individual relations and do not yield predictions as to how a category might be referred to that encompasses a range of spatial relationships grouped together. Identifying systematic patterns in such references will shed more light on the relation between linguistic and non-linguistic spatial direction concepts. For this purpose, we combine the category construction paradigm using the CatScan tool (Klippel et al., 2013) with Cognitive Discourse Analysis (CODA, Tenbrink, in press) by eliciting unconstrained verbalizations in carefully constrained settings, and analyzing the linguistic data systematically with respect to relevant linguistic features. This concerns not only directly elicited labels for categories but also explicit metacognitive statements about how the grouping was performed, so as to shed more light on principles that participants draw upon for the non-linguistic categorization task.

In a nutshell, our study asks (1) whether spatial relationships between objects shown in a realistic scene will be grouped according to known linguistic principles or in other ways, (2) what kinds of linguistic labels are used for categories that were identified non-linguistically, and (3) what kinds of principles people draw upon and verbalize when asked about the non-linguistic grouping task.

\section{Our study: Linguistic and non-linguistic categorization of spatial relations}

We examine the non-linguistic categorization of spatial relations and its interrelation with verbal expression, employing a framework we previously developed for analysis of humans' intuitive spatio-temporal concepts (Klippel et al., 2013). The central component is CatScan, a software we designed to administer category construction experiments (Medin et al. 1987) in which the participant has to sort icons into groups (Figure 1). Neither the groups nor their number are predetermined and the participant is instructed to group the icons based on how similar they are (details in Section 3.3). The software is designed to be compatible with the Amazon Mechanical Turk (AMT) $)^{5}$ crowdsourcing environment. By employing AMT, the challenge of recruiting an adequate participant pool is reduced significantly. AMT has gained widespread recognition in the scientific community with its demonstrated reliability, efficiency, and comparability with lab experiments. Additionally, research on the demographics of AMT workers has shown that general population characteristics are better reflected compared to classic on-campus lab experiments (Ross et al., 2010).

In our studies, a reference object $R$ and a target object $T$ were positioned on a table. Since we were aiming for realistic scenarios, scenes were shown either in a photograph, or in a rendered image of a $3 \mathrm{~d}$ scene model. $R$ and $T$ were either a blue and a yellow ball, respectively, or two mugs of different colors, yielding three different conditions. Figure 2 shows the three types of stimuli used in the study.

\footnotetext{
5 https://www.mturk.com/mturk/
} 


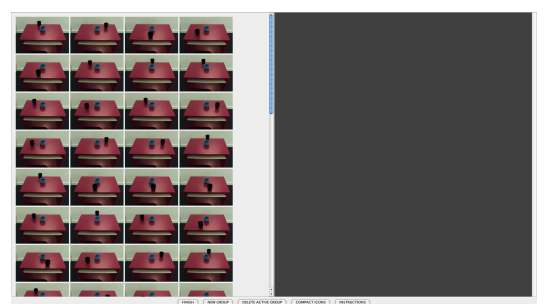

(a)

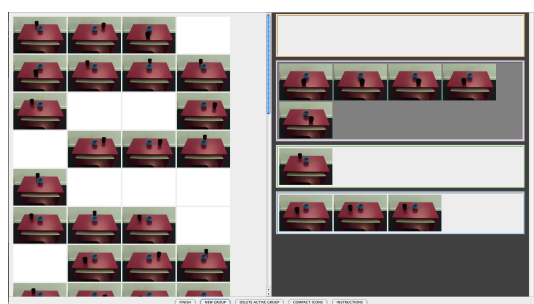

(b)

Fig. 1. (a) Screenshot of the CatScan interface at the beginning of the main study. (b) Screenshot of the interface of an ongoing mock-up trial.

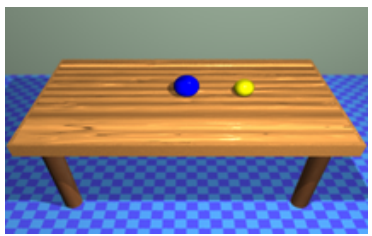

(a)

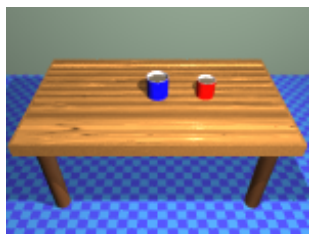

(b)

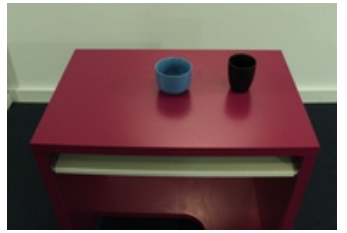

(c)

Fig. 2. (a) Yellow and blue ball in $3 \mathrm{~d}$ model rendering. (b) Red and blue mug in $3 \mathrm{~d}$ model rendering. (c) Black and blue mug in photograph.

\section{$3.1 \quad$ Stimuli}

For each condition (balls/mugs in model, mugs in photograph), 80 images were created. The model-based images were rendered using POV-ray $\sqrt{6}$. The photographs were created by manipulating a physical scene and taking images of different configurations of reference object $R$ and target object $T$ while keeping all camera settings identical. In all images, $R$ was a blue object (mug or ball, accordingly), while $T$ had different colors (yellow ball, red mug, black mug). All mugs were displayed without a handle to avoid effects of handle position. Both objects were placed on a table. The table was placed in the scene such that the camera was oriented towards the center of the table surface, looking down onto the table. As Figure 2 shows, the different types of stimuli were presented from the same perspective horizontally. The camera was always positioned in front of the table with a $90^{\circ}$ angle. Vertical camera position and field of view were slightly varied. $R$ (blue mug/ball) was placed exactly in the center of the table surface. $T$ (yellow ball, red mug, black mug) was placed onto the table with equal distance to $R$ in all images of the same image type. The angle increases in steps of $4.5^{\circ}$.

The effects of differences in stimulus presentation will be subject to further study, but are of no relevance for the work presented here. Thus, all three conditions are analyzed jointly for current purposes.

\footnotetext{
${ }^{6}$ http://www $\cdot$ povray.org/
} 


\section{$3.2 \quad$ Participants}

We recruited 59 participants using AMT. Of these participants, 16 were excluded based on responses to the follow-up verbal task: We excluded participants who reported that they grouped the images based on criteria other than direction (e.g. distance or randomly) or whose category descriptions were inconsistent with the labels such that they indicated contradictory directions. We also excluded participants who created several categories for exactly the same direction and participants who provided incomplete systems, for example using seven cardinal directions $S, S W, W, N W, N, N E, E$, but lacking $S E$. Although the latter may in fact be a legitimate non-linguistic grouping, inspection of the individual cases showed that most of these cases were signs of carelessness or difficulty with the task. In order to be consistent, we removed all participants with incomplete systems. Of the remaining 43 participants, 21 were female and 22 male. The average age was 33.07 (maximum age: 60 , minimum age: 19 ).

\subsection{Procedure}

Individual studies were posted to AMT's webpage. AMT workers were given a unique participant number and instructed to download and run CatScan. Participants were required to enter their demographic information (age, gender, native language, and educational background) and read the study instructions which introduced the basics of the study. To ensure that the participants read and understood the instructions, they were only allowed to proceed after a certain time and had to answer a question about the instructions. In a warm-up task, participants were then acquainted with the interface and the idea of category construction by sorting animals into groups. In the main study, all 80 images were initially displayed in the left panel on the screen. Participants had to sort the images into categories they created on the right panel of the screen (see Figure 1 for a mock-up trial). Once all images were sorted into the categories, participants had to do a follow-up task in which they were presented with the groups they created, one group at a time, and asked to provide a short label (no more than five words) and a detailed description providing reasons for their categories. In addition, they had to select the most representative image for every group they had created. Upon the completion of the second part, CatScan generated a zip file that participants then had to upload to AMT. The zip file contained log files, grouping results, and linguistic descriptions.

\section{Results}

\subsection{Analysis of Segmentation Types}

Based on the verbal labels and descriptions provided by the participants, we annotated the groups created for basic segmentation types: we annotated whether participants divided the relations into four cones, four quadrants, eight cones, or segmented directions in some other way. In two cases, participants did not 
provide descriptive labels (e.g. numbering categories). For those participants, the detailed descriptions were considered for this analysis. In the following, we describe the different segmentation types and give examples for each type.

We counted as 4-cone segmentation all cases where directions were split into exactly four groups, typically labeled with simple linguistic direction terms coinciding with the four prototypical axes of $0^{\circ}, 90^{\circ}, 180^{\circ}$ and $270^{\circ}$. Example 1 shows a typical set of labels for participants using this segmentation. A 4quadrant segmentation was identified when exactly four categories with labels using combined linguistic direction terms were created, as in Example 2, An 8 -cone segmentation was identified when exactly 8 categories with labels using simple and combined linguistic direction terms were created, as in Example 3 .

(1) South vs. West vs. East vs. North (P 12100022)

(2) Upper Left Quadrant vs. Upper Right Quadrant vs. Lower Left Quadrant vs. Lower Right Quadrant (P 12020036)

(3) In Front vs. In Back vs. To the Left vs. To the Right vs. Diagonal Back Right vs. Diagonal Front Right vs. Diagonal Back Left vs. Diagonal Front Left (P 12100009)

Finally, all cases that did not match any of the segmentation types described above were counted as Other. These were half plane-based segmentations, segmentations that conflated opposite directions into one category, and full clock segmentations creating 12 categories according to the hour positions of the clock.

We compared the results of this verbal-label based analysis to a clustering of the non-verbal segmentation behavior in order to verify whether these were indeed coherent segmentation types. Similarity values for each pair of participants were derived by summing up over the pairs of icons that were placed in the same group by one but not the other participant. Ward's method was then used to cluster participants based on these similarity values. Table 1 shows the relationship between segmentation types identified on the basis of verbal as opposed to non-verbal (clustering) data. When two clusters are assumed, all quadrant segmentations fall neatly into one cluster, while all 4-cone segmentations fall into the other cluster. The 8-cone segmentations are divided between both clusters. Finer segmentation with 4 clusters separates the 4-cone segmentations from the 8-cone segmentations, and yields a separate cluster of 3 segmentations annotated as Other. Even when allowing a larger number of clusters, the four deviant 8-cone segmentations remain in one category with with the 4-quadrant segmentations. Closer inspection of individual participants' data showed that this was due to them not being true cone segmentations, but rather form a separate segmentation type: an extension of 4-quadrant segmentations with additional narrow categories for each boundary axis between quadrants. Thus, these four cases will be subsumed under the category Other in the following tables.

In summary, we verified three major segmentation types, 4-cluster, 4-cone, and 8-cone through combining linguistic and non-linguistic analysis. The large proportion of segmentations subsumed under Other shows that there is great variability with respect to segmentation types. For the remainder of this paper, our discussion mainly focuses on the 26 participants who conformed to one of 
the main patterns, since these are theoretically the most interesting. The fact that there are further possibilities and a wide range of individual creativity does not call the existence of the main types into question. Further analysis of the less frequent segmentation types would be highly interesting in order to gain insights into the full range of possibilities of human category formation. As this would require further data in order to obtain a sufficient number of cases for each of the respective categories, it is left for future research.

Table 1. Correspondence of segmentation types derived from analysis of verbal data with clusters of similar participant behaviour from the grouping task.

\begin{tabular}{l|rr|rrr|rrrr}
\hline & \multicolumn{2}{|c|}{ Clusters } & \multicolumn{4}{r|}{ C Clusters } & \multicolumn{3}{|r}{ Clusters } \\
\hline & 1 & 2 & 1 & 2 & 3 & 1 & 2 & 3 & 4 \\
\hline $4 \mathrm{C}$ & 0 & 9 & 0 & 9 & 0 & 0 & 0 & 9 & 0 \\
$4 \mathrm{Q}$ & 11 & 0 & 11 & 0 & 0 & 11 & 0 & 0 & 0 \\
$8 \mathrm{C}$ & 4 & 6 & 4 & 6 & 0 & 4 & 6 & 0 & 0 \\
Other & 7 & 6 & 4 & 6 & 3 & 4 & 4 & 2 & 3 \\
\hline
\end{tabular}

\subsection{Analysis of verbalization types}

In order to determine how the identified segmentation types relate to linguistic categories, we annotated which system of direction terms was used in the category labels. Four main verbalization types were identified: horizontal projective terms describing lateral and frontal (i.e., horizontal) axes on a plane (Example 4), vertical projective terms denoting vertical relationships (Example 5), clock terms which use the hour or minute hand of a clock (Example 6), and cardinal directions, i.e., compass terms (Example 7). Other terms, such as angles or mixed verbalizations, were categorized as Other.

(4) to the right of the blue mug vs. in front of the blue mug vs. to the left of the blue mug vs. behind the blue mug (P 12040018)

(5) To the left vs. Right side vs. Bottom group vs. Top group (P 12100013)

(6) 12:00 to 2:59 vs. 3:00 to 5:59 vs. 6:00 to 8:59 vs. 9:00 to 11:59 (P 12090003)

(7) South vs. West vs. East vs. North (P 12100022)

Table 2 shows the number of participants using the different types of verbalization by segmentation type. Standard projective terms are more frequent than other systems. Overall, the results show that preferences for different segmentation strategies are fairly evenly distributed. However, projective terms are particularly frequent in the Other category, indicating that they are flexibly applicable to many different segmentation types.

Accordingly, there is no one-to-one correspondence between segmentation types and verbalizations. The two most frequent types, 4-cone and 4-quadrant, occur with all major verbalization types, and the 6 instances of 8-cone segmentation occur with all verbalization types except for clock verbalization. 
Table 2. Frequency of the different verbalization types by segmentation type.

\begin{tabular}{l|r|r|r|r|r} 
& 4 Cones & 4 Quadrants & 8 Cones & Other & Total \\
\hline Horizontal Proj. & 3 & 4 & 2 & 7 & 16 \\
Vertical Proj. & 1 & 2 & 1 & 6 & 10 \\
Clock & 2 & 2 & 0 & 1 & 5 \\
Cardinal & 3 & 1 & 3 & 0 & 7 \\
Other & 0 & 2 & 0 & 3 & 5 \\
\hline Total & 9 & 11 & 6 & 17 & 43
\end{tabular}

\subsection{Verbal Analysis of Category Structure}

Going beyond the category labels, we performed an analysis of the verbal descriptions participants gave to clarify the rationale behind their categorization decisions. We analyzed these metacognitive statements for content and linguistic markers indicating prototype versus boundary conceptualization. As Tenbrink (in press) argues, language provides humans with a network of options, allowing them to refer to a given situation in one of several possible ways. While speakers may not even be aware of those choices, they give an insight into the underlying conceptual perspective (Schober, 1998). For example, referring to the same spatial configuration as either "the blue cup is in the bottom-left quadrant" or "the blue cup is to the south-west of the red cup" construes the cup as being contained in a region in the first case, while it is construed as being positioned in a certain direction in the second case. Each kind of expression highlights a different aspect of the situation, thus allowing the conclusion that this aspect is relevant to the way the speaker perceives the situation.

In our analysis, in order to avoid distortions due to different verbosity or repetitiveness of answers, we did not count individual occurrences of markers, but rather the number of participants who used a type of marker at least once.

Markers of Boundary-based Conceptualization We identified the following markers for boundary-based conceptualization in the data:

Dividing: On the content level, many participants explicitly mentioned dividing space into quadrants or segments (see Example 8). This is a very clear indicator for boundary-based conceptualization. We counted all participants as explicitly mentioning a dividing strategy if they at least once used a verb with the semantics of dividing (divide/ split/ segment/ separate) in relation to the space around the reference object $8 \mathrm{a}$ or the spatial relations $8 \mathrm{~b}$.

Boundaries: Some participants made this even more explicit by mentioning specific boundary lines, angles or positions (see Example 9p. We counted participants as explicitly mentioning boundaries if they at least once used a between relationship to explain which images were put in a category $9 \mathrm{a}$, or if they mentioned drawing imaginary boundary lines $(9 \mathrm{~b})$, or when they described specific spatial positions or angles serving as an excluding criterion (9c) for the category. 
Containment: Finally, a linguistic marker of boundary-based conceptualization was describing the location of the target as a containment relation with respect to a region (see example 10) using prepositions such as in (10a), within (10b), or on (10c) and a region term such as area (10c) or quadrant (10a). While a containment relation does not per se exclude prototypes or vague boundaries, we consider it to be indicative of boundary conceptualization, as it constitutes the alternative to a projective relation which emphasizes the aspect of directionality, as usually depicted by an arrow, and consistently shows prototype effects (Crawford et al. 2000, Gapp, 1995, Hayward and Tarr, 1995, Huttenlocher et al., 1991; Vorwerg and Tenbrink 2007, Zimmer et al., 1998). The containment relation, on the other hand, emphasizes the aspect of containment in a region, and thus de-emphasizes any gradual differences within the region.

(8) Explicit mention of dividing into quadrants/segments

a. I divided the area into a quadrant plane (P 12080029)

b. using the blue ball as the center, I divided them into quarters (like the hours on a clock face). (P 12020022)

(9) Mentioning of boundaries

a. They were all between 3:00 and 6:00 (P 12020022)

b. I made an imaginary line on the table both along the length and width going through the blue mug and then asked whether or not it would be in the furthest left quadrant. (P 12080018)

c. Whenever the yellow ball was below the blue ball and less than $45^{\circ}$ to either side I considered it to be on the bottom. (P 12020023)

(10) Containment relation

a. The yellow balls are in the northwest quadrant of the table with regards to blue being the center. (P 12020026)

b. with the blue mug as the 0 on the coordinate axis., All of the black mugs were within the bottom right quadrant of the coordinate planes in relation to the blue mug. (P 12080005)

c. Red cup is located on the area I called lower right quadrent. (P 12040016)

Markers of Prototype-based Conceptualization We identified the following markers of prototype-based conceptualization in the data:

Direction: We counted participants as explicitly mentioning direction if they either mentioned direction as a categorization criterion (Example 11a), or used the term "direction" to explicitly mark the spatial relation between the target object $T$ and the reference object $R$ as a direction (Example 11b).

Projection: While boundary conceptualization is characterized by containment relations (see above), prototype conceptualization is characterized by projective relations. We counted participants as using projective relations if they expressed a projective relation between $T$ and $R$ at least once. We did not consider relations between an area and $R$, only those which located the target object $T$ itself. In 
the data, projective relations were mainly realized as prepositions or preposition groups (Example 12).

Vagueness: While boundary-based concepts tend to be crisp, prototype-based concepts are by definition vague. This can be expressed by vagueness markers such as mostly, roughly, or approximately (Example 13). This was scored if participants used a vagueness marker to modify the location of $T$ at least once, either by directly modifying a projective term 13a with $T$ as the locatum, or by elaborating on such a relation using a vagueness marker $13 \mathrm{~b}$ or a vague exclusion criterion (13c). In contrast to the exclusion criteria which are defining for boundary-based conceptualization, vague exclusion criteria do not specify a concrete line or angle or point. Rather, they specify some criterion which is vaguely tied to a direction or distance relation to an environmental feature.

Comparatives: Another way to indicate vagueness of boundaries is to use comparatives and superlatives when describing the location of $T$ (Example 14), for example by saying that it is closest to a certain prototype, or closer to it than to another (14a), or by using an environmental feature to serve as a prototype, and using relative closeness to this feature to define directions $(14 \mathrm{~b})$.

(11) Direction

a. I decided to group by direction. (P 12020030)

b. Any yellow ball that was facing the North East direction away from the blue ball was considered for this group. (P 12020024)

(12) Projective relations

a. Above the blue mug slightly angled ( $\mathrm{P}$ 12080020)

b. If the black mug was almost straight to the left of the blue mug (P 12080012)

(13) Vagueness

a. any picture where the black mug was in approximately a west, west-southwest, or west-northwest position (P 15998256)

b. Above the blue mug slightly angled ( $\mathrm{P} 12080020)$

c. I chose icons that had the black mug to the right of the blue mug, but not too far up or down (P 12080025)

(14) Comparatives

a. I picked the mugs in this group that were the westernmost mugs in relation to the blue mug. (P 12080008)

b. the black mug was closer to the front of the desk ( $\mathrm{P}$ 12090005)

Table 3 shows the total number of participants using either no markers at all, or at least one marker of boundary and/or prototype conceptualizations in their detailed category descriptions in relation to the segmentation types. As the table shows, almost all participants used markers of at least one of the conceptualizations (41 out of 43). All participants who segmented according to quadrants used boundary markers, while only few of them used prototype markers. Conversely, participants who used cone segmentation predominantly 
Table 3. Number of participants using markers of boundary and/or prototype conceptualization in their group descriptions, grouped by segmentation types.

\begin{tabular}{l|r|r|r|r|r} 
& 4 Cones & 4 Quadrants & 8 Cones & Other & Total \\
\hline none & 0 & 0 & 0 & 2 & 2 \\
prototype & 4 & 0 & 6 & 11 & 21 \\
boundary & 1 & 8 & 0 & 1 & 10 \\
both & 4 & 3 & 0 & 3 & 10 \\
\hline Total & 9 & 11 & 6 & 17 & 43
\end{tabular}

Table 4. Usage of the different markers of prototype and boundary-based conceptualization in their group descriptions by segmentation types. Each participant may have used none, one, or several types of markers, therefore column-totals do not represent number of participants.

\begin{tabular}{l|r|r|r|r|r} 
& 4 Cones & 4 Quadrants & 8 Cones & Other & Total \\
\hline divide & 2 & 2 & 0 & 1 & 5 \\
boundaries & 2 & 4 & 0 & 3 & 9 \\
containment & 2 & 9 & 0 & 3 & 14 \\
\hline Total Boundary & 6 & 15 & 0 & 7 & 28 \\
\hline & & & & & \\
\hline direction & 0 & 0 & 1 & 2 & 3 \\
projection & 6 & 3 & 6 & 12 & 27 \\
vagueness & 4 & 0 & 5 & 8 & 17 \\
comparative & 0 & 1 & 1 & 3 & 5 \\
\hline Total Prototype & 10 & 4 & 13 & 25 & 52 \\
\hline
\end{tabular}

used prototype markers, a tendency which is more strongly expressed for the 8-cone segmentation which co-occurs only with prototype markers. For the 4-cone segmentation, either prototype or a mix of prototype and boundary markers were found, while one participant used boundary markers only. Although these are clear tendencies, the results show that there is no strict one-to-one correspondence of prototype vs. boundary conceptualization to segmentation type: in all segmentation types except for the 8-cone segmentation, there are participants who used markers of both conceptualizations.

Table 4 shows a more detailed view of the occurrence of markers of prototypeand boundary-based conceptualization with the different segmentation types. The clearest indicators of prototype-based conceptualization are vagueness and projective terms, while comparatives and explicit mention of direction play virtually no role due to their rare occurrence. Quadrant-based segmentation coincided with some projective relations and one comparative, but none of the other features. The most dominant indicator of a quadrant segmentation is the use of a containment relation to describe the location of the target object. This marker only co-occurred very rarely with cone-based segmentation. The explicit mention of boundaries was only slightly more frequent for quadrant segmentation than for the other types, and explicit mention of dividing lines was fairly infrequent overall, and also not limited to quadrant-based segmentation. 


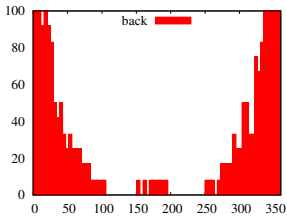

(a) BEHIND

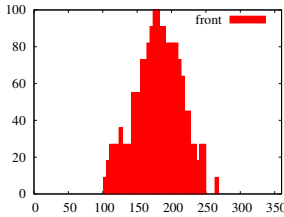

(b) Front

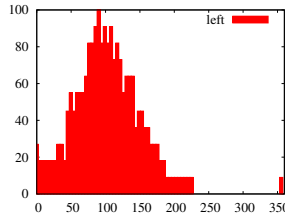

(c) LEFT

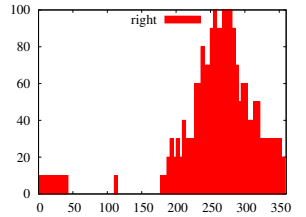

(d) Right

Fig. 3. Structure of simple term categories. X-axis represents angles starting with 0 at prototypical BEHIND, and increasing counter-clockwise. Y-axis represents percentage of occurring groups of the respective category containing the image with that angle.

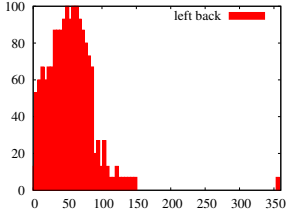

(a) LEFT BEHIND

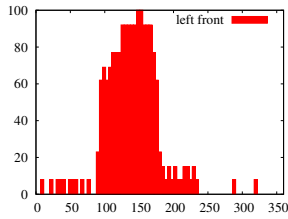

(b) LEFT FRONT

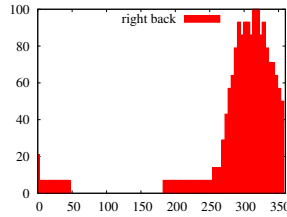

(c) Right BEHIND

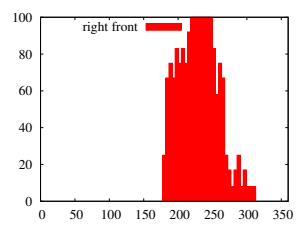

(d) Right FRONT

Fig. 4. Relative frequency of occurrence of each angle in complex term categories.

\subsection{Visual Analysis of Category Structure}

Based on the analysis of verbalization types, we conclude that different types of direction terms such as left,right,front,back or north,south,east,west do not correspond to different segmentation types. On the other hand, the analysis of markers for conceptualizations suggests that the central axes $-0^{\circ}, 90^{\circ}, 180^{\circ}$ and $270^{\circ}$ - play a crucial role for both quadrant and cone segmentation. To further verify this claim, we combined data from all types of direction terms across all types of segmentation and compared the angular spread of those categories which are expressed by simple direction terms such as left or west (simple term categories) with that of categories which are expressed by complex direction terms such as left-front or southwest (complex term categories). For the sake of simplicity, we named the directions according to horizontal projective terms, though we use small caps spelling to indicate that these are the underlying (linguistic) concepts, and not the actual verbalizations.

Figure 3 shows that for simple term concepts, there is a small frequency peak around the established prototypes of $0^{\circ}, 90^{\circ}, 180^{\circ}$ and $270^{\circ}$, and gradually decreasing frequency of inclusion with further distance from the prototype. The extremes of all concepts except for FRONT lie about $200^{\circ}$ apart, confirming the large variance of boundaries. FRONT shows the same overall pattern, although its maximal range is slightly narrower than that of the other concepts. This indicates that participants associated a wide variety of non-linguistic categories with simple term linguistic concepts, all centered around the prototype, but varying in extension depending on the segmentation strategy used.

On the other hand, the complex term concepts show little boundary variation (Figure 4) with a high plateau and a steep drop approximately at the central 
axes, supporting the assumption that those combined term concepts are mostly defined by their boundaries, and therefore cannot be appropriately used for many different segmentation strategies. The variation within the plateau is most probably due to the influence of the 8-cone segmentation, which seems to use additional intermediate prototypes.

\section{Discussion and Conclusion}

We have presented a category construction study in which participants sorted images of object configurations into groups of similar spatial relationships. Our findings reveal that the available space was segmented mostly into either four cones, four quadrants, or eight cones. These segments did not correspond directly to any specific types of verbal labels (horizontal or vertical projective, clockbased, cardinal) that participants assigned to their categories; in that sense, no simple one-to-one correspondence between linguistic and non-linguistic categories could be found. However, only four-cone segments were represented by simple terms such as left or north, reflecting the fact that four basic spatial directions can easily be expressed in language (e.g., using sets such as left, right, front, behind or the compass terms). Other concepts that do not directly correspond to this kind of linguistic set, such as a more fine-grained segmentation of space (into 8 cones) or a quadrant-based segmentation, require more complex spatial descriptions. This matches well with the literature on complex and non-standard spatial relationships (Vorwerg, 2003, Zimmer et al., 1998).

More surprisingly, our linguistic analysis of metacognitive strategy verbalizations revealed that the different segmentation types related to fundamentally distinct categorization concepts. Cone-based segmentation was associated with prototypes, and quadrant-based segmentation with boundaries. Linguistically, prototype-based categories were almost exclusively verbalized as a projective relation between two objects, while boundary-based categories were mostly verbalized as a containment relation between an object and a region. The visual analysis of grouping patterns confirmed the existence of these two distinct types of categorization in simple vs. combined linguistic term concepts. Both were dominated by the four major axes of $0^{\circ}, 90^{\circ}, 180^{\circ}$, and $270^{\circ}$, albeit in different ways: in one case they served as prototypes, in the other as boundaries. The 8-cone segmentation appeared to make use of additional secondary prototypes.

Thus, our results support earlier findings by Crawford et al. (2000); Klippel and Montello (2007) and Huttenlocher et al. (1991) about the dual role of the major axes in different categorization types. However, since their research did not include any analysis of language used to describe these two category concepts, the relationship between verbalization and conceptualization had not been addressed. Our findings show for the first time how categorization relates to two fundamentally distinct types of concepts expressed in spatial language: containment vs. direction. As highlighted by Bateman et al. (2010), these two types of terms are based on ontologically distinct spatial concepts and as a consequence exhibit distinct linguistic patterns. We conclude that rather than reflecting linguistic versus non-linguistic direction categories, prototype and boundary based categorization 
are two separate non-linguistic strategies of dividing space, each with its own suitable verbalization strategy.

\section{Acknowledgements}

Funding by the Deutsche Forschungsgemeinschaft (DFG) for the SFB/TR 8 Spatial Cognition, project I5-[DiaSpace] and the National Science Foundation under grant number \#0924534 is gratefully acknowledged.

\section{References}

Bateman, J., Tenbrink, T., Farrar, S.: The role of conceptual and linguistic ontologies in interpreting spatial discourse. Discourse Processes 44(3), 175-212 (2007)

Bateman, J.A., Hois, J., Ross, R.J., Tenbrink, T.: A linguistic ontology of space for natural language processing. Artif. Intell. 174(14), 1027-1071 (2010)

Bierwisch, M., Schreuder, R.: From concepts to lexical items. Cognition 42, 23-60 (1992)

Carlson, L.A., van der Zee, E. (eds.): Functional features in language and space: Insights from perception, categorization and development. Oxford University Press, Oxford (2005)

Crawford, L.E., Regier, T., Huttenlocher, J.: Linguistic and non-linguistic spatial categorization. Cognition 75(3), 209-235 (2000)

Gapp, K.P.: An empirically validated model for computing spatial relations. In: Proceedings of KI-95. Springer, Berlin, Heidelberg (1995)

Hayward, W.G., Tarr, M.J.: Spatial language and spatial representation. Cognition 55, 39-84 (1995)

Herzog, G.: Coping with static and dynamic spatial relations. In: P. Amsili, M. Borillo, L.V. (ed.) Proc. of TSM'95, Time, Space, and Movement: Meaning and Knowledge in the Sensible World, pp. 47-59 (1995)

Hickmann, M., Hendriks, H.: Static and dynamic location in French and English. First Language 26(1), 103-135 (2006)

Huttenlocher, J., Hedges, L.V., Duncan, S.: Categories and particulars: Prototype effects in estimating spatial location. Psychological Review 98(3), 352 (1991)

Klippel, A., Montello, D.R.: Linguistic and nonlinguistic turn direction concepts. In: Spatial Information Theory. Springer, Berlin (2007)

Klippel, A., Wallgrün, J.O., Yang, J., Mason, J.S., Kim, E.K., Mark, D.M.: Fundamental cognitive concepts of space (and time): Using cross-linguistic, crowdsourced data to cognitively calibrate modes of overlap. In: COSIT. pp. 377-396 (2013)

Levinson, S.C., Kita, S., Haun, D.B., Rasch, B.H.: Returning the tables: Language affects spatial reasoning. Cognition 84, 155-188 (2002)

Li, P., Gleitman, L.: Turning the tables: Language and spatial reasoning. Cognition 83, 265-294 (2002)

Logan, G.D., Sadler, D.D.: A computational analysis of the apprehension of spatial relations. In: Bloom, P., Peterson, M., Nadell, L., Garrett, M. (eds.) Language and Space, pp. 493-529. MIT Press (1996) 
Medin, D.L., Wattenmaker, W.D., Hampson, S.E.: Family resemblance, conceptual cohesiveness, and category construction. Cognitive Psychology 19(2), 242-279 (1987)

Mervis, C.B., Rosch, E.: Categorization of natural objects. Annual Review of Psychology 32, 89-115 (1981)

Renz, J., Nebel, B.: Qualitative spatial reasoning using constraint calculi. In: Aiello, M., Pratt-Hartmann, I.E., van Benthem, J.F.A.K. (eds.) Handbook of Spatial Logics, pp. 161-215. Springer (2007)

Ross, J., Irani, L., Silberman, M.S., Zaldivar, A., Tomlinson, B.: Who are the crowdworkers? Shifting demographics in mechanical turk. In: CHI EA 2010 Extended Abstracts on Human Factors in Computing Systems. pp. 2863-2872 (2010)

Schober, M.F.: Different kinds of conversational perspective-taking. In: Fussell, S.R., Kreuz, R.J. (eds.) Social and Cognitive Psychological Approaches to Interpersonal Communication. Lawrence Erlbaum, Mahwah, NJ (1998)

Smith, E.E., Medin, D.L.: Categories and Concepts. Harvard University Press, Cambridge, MA (1981)

Taylor, J.: Linguistic Categorization. Clarendon, Oxford (1989)

Tenbrink, T.: Space, time, and the use of language: An investigation of relationships. Mouton de Gruyter, Berlin (2007)

Tenbrink, T.: Reference frames of space and time in language. Journal of Pragmatics 43(3), $704-722$ (2011)

Tenbrink, T.: Cognitive discourse analysis: Accessing cognitive representations and processes through language data. Language and Cognition (in press)

Vorwerg, C.: Use of reference directions in spatial encoding. In: Spatial Cognition III: Routes and Navigation, Human Memory and Learning, Spatial Representation and Spatial Learning, pp. 321-347. Springer, Berlin (2003)

Vorwerg, C., Tenbrink, T.: Discourse factors influencing spatial descriptions in English and German. In: Barkowsky, T., Knauff, M., Ligozat, G., Montello, D. (eds.) Spatial Cognition V: Reasoning, Action, Interaction, pp. 470-488. Springer, Berlin, Heidelberg (2007)

Whorf, B.: Language, thought, and reality: Selected writings of Benjamin Lee Whorf. The M.I.T. Press, Cambridge, MA (1956), (Edited by John Carrol)

Zimmer, H., Speiser, H., Baus, J., Blocher, A., Stopp, E.: The use of locative expressions in dependence of the spatial relation between target and reference object in two-dimensional layouts. In: Freksa, C., Habel, C., Wender, K. (eds.) Spatial cognition: An Interdisciplinary Approach to Representing and Processing Spatial Knowledge, Lecture Notes in Computer Science, vol. 1404, pp. 223-240. Springer, Berlin / Heidelberg (1998)

Zwarts, J.: Vectors across spatial domains: from place to size, orientation, shape, and parts. In: van der Zee, E., Slack, J. (eds.) Representing Direction in Language and Space, pp. 39-68. Oxford University Press, Oxford (2003) 